\title{
Condición física, composición corporal y rendimiento académico en niños/as con sobrepeso/obesidad
}

\section{Fitness, body composition and academic achievement in overweight/obese children}

\author{
Miguel Ángel Ortega Jiménez \\ Grupo de Investigación HUM 727 - Universidad de Granada, Granada (España)
}

\begin{abstract}
Resumen: Objetivos: Se ha sugerido que la condición física y el sobrepeso están relacionados con el rendimiento académico. Este estudio examinó la asociación entre indicadores de masa corporal, masa grasa y condición física sobre el rendimiento académico, en niños/as preadolescentes con sobrepeso/ obesidad. Método: 19 niños/as preadolescentes con sobrepeso/obesidad. Estudio transversal en el que se evaluaron el IMC, IMG, la capacidad aeróbica (test de $20 \mathrm{~m}$ ), $\mathrm{VO}_{2}$ max mediante un test de laboratorio y estimado indirectamente a partir del test de $20 \mathrm{~m}$, fuerza muscular, velocidad-agilidad y el rendimiento académico, a través de la Batería III Woodcock - Muñoz ${ }^{\mathrm{TM}}$. Resultados: el rendimiento académico total se relacionó con la capacidad aeróbica, el $\mathrm{VO}^{2} \max$, la fuerza del tren inferior y la velocidad-agilidad. La capacidad aeróbica fue asociada con la lectura y destrezas académicas. La fuerza del tren inferior y la velocidad-agilidad con la lectura, las matemáticas y las destrezas académicas. La composición corporal no tuvo asociación. Palabras clave: condición física, masa grasa, obesidad, rendimiento académico.
\end{abstract}

Abstract: Objectives: It has been suggested that physical condition and being overweight are related to academic performance. This study examined the association between indicators of body mass, fat mass and physical condition on academic performance in overweight / obese preadolescent children. Method: 19 overweight / obese preadolescent children. Crosssectional study in which BMI, IMG, aerobic capacity (20 m test), VO2max were evaluated through a laboratory test and indirectly estimated from the $20 \mathrm{~m}$ test, muscle strength, speed-agility and academic performance, through Woodcock III Battery - MuñozTM. Results: Total academic performance was related to aerobic capacity, $\mathrm{VO} 2 \mathrm{max}$, lower body strength and speed-agility. Aerobic capacity was associated with reading and academic skills. Lower body strength and speed-agility with reading, math, and academic skills. Body composition had no association.

Keywords: fitness, fat mass, obesity, academic achievement.

\section{Introducción}

La Organización Mundial de La Salud (OMS) advierte que en las últimas décadas el sobrepeso y la obesidad infantil se ha convertido en uno de los mayores problemas de nuestra sociedad (Ogden, 2014). En 2010 hubo unos 42 millones de niños/as con sobrepeso en todo el mundo, de los que casi 35 millones vivían en países en desarrollo (OMS, 2015). Estos datos están llegando a alcanzar valores epidémicos en la mayoría de los países desarrollados convirtiéndose en un problema de salud pública. España, junto con Malta y Sicilia, lidera el ranking europeo de sobrepeso/obesidad en niños/as de 7 a 11 años según la Asociación Europea para el estudio de la Obesidad (EASO, 2015).

Cada vez más estudios muestran la eficacia del ejercicio físico como una de las estrategias más útiles en la prevención y mejora del sobrepeso/obesidad (Donnelly, 1996; Hills, 2011). Algunos estudios relacionan el sobrepeso con un bajo rendimiento académico en niños/as(Castelli, 2007; Roberts, 2010; Welk, 2010). Estos estudios relacionan el índice de masa corporal (IMC) y algunos parámetros de condición física con el

Dirección para correspondencia [Correspondence address]: Miguel Ángel Ortega Jiménez. E-mail: maortegaj@outlook.com rendimiento académico, extrayendo conclusiones de asociación positiva entre condición física y rendimiento académico. Otro estudio transversal también muestra la existencia de la correlación entre grasa corporal y rendimiento académico (Davis, 2011), sin embargo, la medida del rendimiento académico en Educación Primaria en España tiene solo 5 valores categóricos, siendo una medida muy poco precisa y sensible.

La condición física es un importante marcador de salud, tanto en edades más tempranas como en las más avanzadas (Ardoy, 2011; Ortega, 2008). Son numerosos los beneficios que una buena condición física proporciona a la salud física y mental (Ruiz, 2009). Un importante número de estudios constatan que la condición física también supone un papel importante en la salud del cerebro y en el rendimiento académico en niños/as y adolescentes (Ardoy, 2014;Donnelly, 2011;Esteban-Cornejo, 2014;Lees, 2013;Pontifex, 2011;Torrijos-Nino, 2014). Estos estudios también encuentran una relación positiva entre la capacidad aeróbica y el rendimiento académico global, matemáticas y lectura (Castelli, 2007 \#23). Esto muestra la implicación directa entre actividad física y rendimiento escolar.

Dado que, desde nuestro conocimiento, existe poca evi- 
dencia científica sobre la asociación entre los componentes de la condición física relacionada con la salud y grasa corporal con el rendimiento académico, el planteamiento de este estudio pretende ampliar dicha evidencia científica en este sentido. El objetivo fue examinar la asociación entre indicadores de masa corporal, masa grasa y condición física sobre el rendimiento académico en niños/as preadolescentes con sobrepeso/obesidad.

\section{Método}

\section{Diseño}

Este trabajo forma parte del proyecto ActiveBrains sobre el cerebro, el rendimiento cognitivo, el rendimiento académico y la salud física y mental en nińos/as preadolescentes con sobrepeso/obesidad. Los datos que se presentan han sido obtenidos en la valoración inicial por lo que se trata de un estudio transversal. Dicho proyecto fue aprobado por el Comité de Ética en investigación Humana de la Universidad de Granada (Referencia: 848, febrero 2014).

\section{Participantes}

Un total de 19 participantes (11 chicas, 10,18 $\pm 0,73$ años) procedentes de la provincia de Granada formaron parte del presente estudio, que se realizó durante el curso académico 2014/2015. Previamente las familias fueron informadas y se les solicitó un consentimiento informado.

La captación se hizo a través de la Unidad de Pediatría del Hospital Universitario Virgen de las Nieves de Granada (España) y el contacto con los directores de los colegios públicos y privados. Paralelamente se dio difusión a través de prensa y radio.

Todos los niños/as y niñas que participaron en el estudio se ajustaron a los siguientes criterios de inclusión:

1. Estar en el estadio I o II de Tanner (aproximadamente entre 8 y 11,5 años) (Tanner, 1976)

2. Tener sobrepeso u obesidad definidas siguiendo los puntos de corte de índice de masa corporal (IMC) estratificados por edad y sexo (Cole, 2012).

3. No tener ninguna discapacidad física que impida la práctica de actividad física (informe médico).

4. No estar tomando medicamentos que influyan sobre el sistema nervioso central.

5. No tener trastorno por déficit de atención e hiperactividad (TDAH).

6. Ser diestro.

7. No usar ortodoncia o puentes metálicos dentales.

8. Para las niñas, no presentar menarquia.

\section{Mediciones}

Composición Corporal

El peso corporal $(\mathrm{kg})$ fue medido utilizando una báscula de alta precisión (SECA 869, Hamburgo, Alemania, precisión $100 \mathrm{~g})$. La altura (cm) fue medida usando un estadiómetro (SECA 213, Hamburgo, Alemania) con aproximación de 0,1 $\mathrm{cm}$. El índice de masa corporal (IMC) fue calculado como peso $(\mathrm{kg})$ /altura $(\mathrm{m})^{2}$. El perímetro de cintura $(\mathrm{cm})$ fue medido con una cinta métrica no elástica (SECA 200; rango, 0-150 cm; precisión $1 \mathrm{~mm}$ ) en el nivel más estrecho, entre el borde del costal inferior $\left(10^{\mathrm{a}}\right.$ costilla) y la cresta iliaca, al final de una espiración normal y sin que la cinta presionara la piel. Se utilizó la absorciometría de rayos X de energía dual o densitometría ósea (DXA, Hologic Explorer scanner) utilizando una versión pediátrica del programa informático QDRExplorer (versión 12.4, Bedford, MA, EE. UU.) para calcular el índice de masa grasa $(\mathrm{kg}) /$ altura $(\mathrm{m})^{2}$. El protocolo de medición se realizó siguiendo estudios precedentes (Gracia-Marco, 2012).

\section{Características sociodemográficas}

Nivel socioeconómico y nivel educativo de los padres

El nivel socioeconómico de la familia se evaluó a través de la Escala de Riqueza Familiar (ERF). Esta escala se basa en cuatro preguntas que reflejan el gasto y el consumo de cada familia (Currie, 1997). La puntuación obtenida se dividió en tres niveles (bajo, medio y alto) (Jimenez Pavon, 2010). Tanto el padre como la madre fueron preguntados por su nivel máximo de estudios para conocer el nivel educativo de ambos. Al igual que la ERF, se categorizó en bajo (ningún nivel de estudios o estudios primarios), medio (estudios secundarios, formación profesional y bachillerato) y alto (estudios universitarios).

\section{Condición Física}

La condición física (capacidad aeróbica, fuerza muscular y velocidad - agilidad) fueron evaluadas a través de la batería ALPHA fitness, www.thealphaproject.net (Ruiz, 2011). La capacidad aeróbica también fue evaluada en laboratorio mediante una prueba de esfuerzo máximo en tapiz rodante.

\section{Fuerza Muscular}

\section{Fuerza de Prensión Manual}

Para medir la fuerza isométrica del tren superior se utilizó el test de fuerza de prensión manual. Se utilizó un dinamó- 
metro con agarre ajustable (TKK 5101 Grip D; Takei, Tokyo Japan) y una regla-tabla para la anchura del agarre de la mano en niños/as de 6 a 12 años. El participante realizó dos intentos con cada mano, de forma alternativa, apretando al máximo hasta mantener al menos 2 segundos sin que el dinamómetro tuviera contacto con el cuerpo. El tamaño del agarre se ajustó para cada participante haciendo según medición de la regla-tabla (Espana-Romero, 2008). Se registraron dos valores por cada mano, se tomó el más alto y para el análisis se utilizó la media de ambas manos. La medida se realizó en kilogramos $(\mathrm{kg})$.

\section{Salto de longitud con pies juntos}

Esta prueba evalúa la fuerza explosiva del tren inferior. Para ello se utilizó una superficie no deslizante, señalizada con una cinta métrica y dos conos que indicaban la línea de partida. Los participantes se colocaban tras la línea de partida con los pies a la altura de los hombros y realizaban un salto horizontal con los pies juntos. Los participantes tomaban contacto con el suelo con los dos pies de forma simultánea y en posición vertical, quedándose estáticos para tomar la medición. Se realizaron dos intentos válidos y se registraron sendas medidas. Para el análisis se tomó el valor más alto.

\section{Velocidad-agilidad}

\section{Test de $4 \times 10 \mathrm{~m}$}

Para medir la velocidad de movimiento, la agilidad y la coordinación se utilizó el test de 4x10 m. La prueba se realizó en una superficie no deslizante. En ella se dibujaron dos líneas paralelas separadas por una distancia de 10 metros. En la línea de salida se colocó una esponja y en la línea opuesta se colocaron 2 esponjas, todas ellas de diferente color. El resultado de la prueba se registra en segundos (seg) medidos con un cronometro e indicando, al menos, un decimal. Se realizan dos intentos con un descanso para recuperar. Se registran los dos intentos y se tomó para el análisis la mejor marca (el menor tiempo).

\section{Capacidad aeróbica o cardiorrespiratoria}

\section{Test de ida y vuelta de 20 m (Course Navette)}

Este test se utilizó para medir la capacidad aeróbica. Para su desarrollo se utilizó una pista polideportiva para marcar una distancia de 20 metros, 4 conos para delimitar la zona, cinta métrica, un $\mathrm{CD}$ con el protocolo del test y un reproductor de CD.

El participante debía desplazarse de una línea a otra situa- das a 20 metros de distancia y hacer un cambio de sentido al ritmo indicado por la señal sonora. Esta señal iba acelerándose progresivamente. La velocidad inicial de la señal fue de $8,5 \mathrm{~km} / \mathrm{h}$, y el incremento de $0,5 \mathrm{~km} / \mathrm{h} / \mathrm{min}$. La prueba finalizaba cuando el participante no era capaz de llegar por segunda vez consecutiva a una de las líneas con la señal de audio o cuando el participante se paraba debido a la fatiga. Se anotaron el número total de vueltas completadas por cada participante. Además, se calculó el consumo máximo de oxigeno $\left(\mathrm{VO}^{2} \mathrm{max}\right)$ siguiendo la ecuación de Leger (Leger, 1988).

Capacidad aeróbica en test de laboratorio

Prueba de esfuerzo máximo en tapiz rodante

La capacidad aeróbica también fue evaluada mediante un analizador de gases (General Electric Corporation) en una prueba de esfuerzo incremental máximo (Ergómetro hp-cosmos) modificada para niños/as (Protocolo Smart modificado) (Davis, 2012). Los participantes caminaron sobre una cinta a una velocidad constante de $4,8 \mathrm{~km} / \mathrm{h}$, aumentando un $1 \%$ la pendiente cada minuto hasta el agotamiento. El $\mathrm{VO}^{2} \max$ ( $\mathrm{ml} / \mathrm{kg} / \mathrm{min})$, la frecuencia cardíaca (latidos/min) y la relación de intercambio respiratorio (RER) se registraron cada 30 segundos. La percepción subjetiva del esfuerzo (RPE) se registró cada 60 segundos mediante la escala OMNI para niños/ as (Utter, 2002).

\section{Rendimiento Académico}

Para la medición del rendimiento académico de utilizó la Batería III Woodcock - Muñoz ${ }^{\mathrm{TM}}$ (rango de 2 a 90 años), traducción de la Batería Woodcock - Johnson III ${ }^{\circledR}\left(\mathrm{WJ}\right.$ III $\left.{ }^{\oplus}\right)$. El test se componen de dos baterías: estándar y extendida. En este estudio se utilizó la Batería Estándar formada por 3 pruebas de lectura, 2 de lenguaje oral, 3 de matemáticas y 3 de lengua escrita. Además se utilizó 1 prueba de conocimiento académico (Ciencias, Ciencias Sociales y Humanidades), incluida en la Batería Extendida.

La administración de la batería de pruebas se realizó de manera ágil y continua, a no ser que el participante necesitara algún pequeño descanso. El test consta de una hoja de respuestas para el alumno y una hoja de puntuación para el examinador. Algunas pruebas con un tiempo establecido requerían la utilización de un cronómetro. El ítem de inicio de cada prueba lo marca el grado escolar en el que se encuentre el participante, siguiendo las indicaciones del test. El anexo V incluye más especificaciones sobre este test.

Tras el registro de las puntuaciones en el software específico (WJ III UN Compuscore and Profiles v 3.1, EE.UU.), se obtuvieron y analizaron los siguientes compuestos:

\footnotetext{
- Rendimiento total
} 
- Lectura

- Matemáticas

- Lenguaje escrito

- Destrezas en cálculo matemático

- Expresión escrita

- Destrezas académicas

- Fluidez académica

- Aplicaciones académicas

- Conocimientos académicos (batería extendida)

\section{Análisis estadístico}

Para el análisis estadístico se utilizó el software informático SPSS (versión 20; SPSS Inc., Chicago, IL). El test de correla- ciones parciales fue utilizado para examinar la relación entre las variables de composición corporal, condición física y rendimiento académico, ajustadas por sexo, edad, nivel socioeconómico familiar y nivel educativo del padre (Castillo, 2011). También se utilizó la regresión lineal para profundizar en la relación existente entre las variables con resultados significativos o con tendencia a la significación, determinando cuanto cambia el rendimiento académico por unidad que incrementan las variables de fitness o grasa.

\section{Resultados}

Las características descriptivas de la muestra de estudio se muestran en la tabla 1.

Tabla 1. Características descriptivas de la muestra de estudio y variables de ajuste.

\begin{tabular}{|c|c|c|c|c|c|}
\hline & $\mathrm{n}$ & Media & DT & Mínimo & Máximo \\
\hline Edad (años) & 18 & 10,18 & 0,73 & 8,94 & 11,81 \\
\hline Peso $(\mathrm{Kg})$ & 18 & 57,68 & 11,23 & 37,50 & 75,60 \\
\hline Talla $(\mathrm{cm})$ & 18 & 144,51 & 7,30 & 127,05 & 158,00 \\
\hline Perímetro de Cintura (cm) & 18 & 85,63 & 10,07 & 73,15 & 106,65 \\
\hline Índice de masa corporal $\left(\mathrm{kg} / \mathrm{m}^{2}\right)$ & 18 & 27,46 & 4,15 & 22,39 & 36,54 \\
\hline Índice de masa grasa $\left(\mathrm{kg} / \mathrm{m}^{2}\right)$ & 18 & 14,06 & 3,42 & 9,67 & 22,21 \\
\hline $\mathrm{VO}^{2} \max (\mathrm{ml} / \mathrm{kg} / \mathrm{min})$ & 18 & 34,48 & 4,64 & 26,00 & 43,50 \\
\hline $\mathrm{VO}^{2} \max ($ Leger et al) $(\mathrm{ml} / \mathrm{kg} / \mathrm{min})$ & 18 & 41,59 & 1,99 & 38,14 & 45,95 \\
\hline Capacidad aeróbica $20 \mathrm{~m}$ (vueltas) & 18 & 14,83 & 7,04 & 5,00 & 33,00 \\
\hline Fuerza Prensión Manual (Kg) & 18 & 16,64 & 4,63 & 9,00 & 28,10 \\
\hline Fuerza Tren inferior (cm) & 18 & 105,22 & 19,18 & 70,00 & 141,00 \\
\hline \multirow[t]{2}{*}{ Velocidad Agilidad 4x10m (seg) } & 18 & 15,09 & 1,39 & 13,00 & 17,48 \\
\hline & & $\mathbf{n}$ & $\%$ & & \\
\hline Sexo, Chicas & & 11 & 61,1 & - & - \\
\hline Escala de Riqueza Familiar & & - & - & - & - \\
\hline Bajo & & 3 & 16,7 & - & - \\
\hline Medio & & 6 & 33,3 & - & - \\
\hline Alto & & 9 & 50,0 & - & - \\
\hline Nivel Educativo del Padre & & - & - & - & - \\
\hline Bajo & & 2 & 12,5 & - & - \\
\hline Medio & & 7 & 43,8 & - & - \\
\hline Alto & & 7 & 43,8 & - & - \\
\hline
\end{tabular}

DT: desviación típica; $\mathrm{VO}^{2} \max$ : consumo máximo de oxígeno.

Las características descriptivas del test Woodcock - Muñoz con los diferentes compuestos de rendimiento académico se muestran en la tabla 2 . 
Tabla 2. Características descriptivas de los diferentes compuestos del test Woodcock - Muńoz.

\begin{tabular}{lccccc}
\hline & $N$ & Media & DT & Mínimo & Máximo \\
\hline Lectura & 18 & 116,00 & 17,58 & 88 & 149 \\
Matemáticas & 18 & 102,78 & 9,38 & 89 & 123 \\
Lenguaje Escrito & 18 & 119,56 & 10,94 & 101 & 140 \\
Conocimientos Académicos (Ciencias sociales, ciencias naturales y humanidades) & 17 & 93,94 & 16,06 & 45 & 115 \\
Aplicaciones Académicas & 18 & 96,44 & 6,97 & 81 & 111 \\
Fluidez Académica & 18 & 110,94 & 11,70 & 89 & 128 \\
Destrezas Académicas & 18 & 129,83 & 19,32 & 102 & 165 \\
Expresión Escrita & 18 & 104,94 & 8,83 & 87 & 119 \\
Destrezas en Cálculo Matemático & 18 & 107,44 & 10,56 & 89 & 128 \\
Aprovechamiento Total & 18 & 114,94 & 13,24 & 91 & 140 \\
\hline
\end{tabular}

DT: desviación típica.

La correlación entre las variables de composición corporal, condición física y rendimiento académico, ajustado por sexo, edad, nivel socioeconómico de la familia y nivel educativo del padre, se muestra en la tabla 3. No se observa ninguna correlación entre las variables de composición corporal (IMC e IMG) y ninguno de los compuestos de rendimiento académico. La fuerza de prensión manual tampoco se relacionó de forma significativa con el rendimiento académico. La capacidad aeróbica, medida con el test de $20 \mathrm{~m}$ se relacionó de manera significativa con la lectura $(r=0,686 ; p=0,020)$, las destrezas académicas $(\mathrm{r}=0,676 ; \mathrm{p}=0,022)$ y el rendimiento total $(\mathrm{r}=0,729 ; \mathrm{p}=0,011)$. Los valores de $\mathrm{VO}^{2} \max$, extraídos del test de ida y vuelta $20 \mathrm{~m}$, se relacionaron significativamente con el lenguaje escrito $(r=0,642 ; p=0,033)$ y rendimiento total $(r=0,640 ; p=0,034)$. De igual modo, esta misma variable
$\left(\mathrm{VO}^{2} \max \right)$ tuvo una relación con tendencia a la significación con respecto a las destrezas académicas $(r=0,594 ; \mathrm{p}=0,054)$. Sin embargo, con los valores de $\mathrm{VO}^{2}$ max obtenidos en laboratorio tan solo se encontró relación significativa con lenguaje escrito $(r=0,616 ; p=0,043)$. La lectura $(r=0,606 ; p=0,048)$, las destrezas académicas $(\mathrm{r}=0,718 ; \mathrm{p}=0,013)$ y el rendimiento total $(\mathrm{r}=0,704 ; \mathrm{p}=0,016)$ también se relacionaron significativamente con la fuerza del tren inferior, así mismo esta misma variable tuvo una tendencia a la significación en relación con las matemáticas $(\mathrm{r}=0,586 ; \mathrm{p}=0,058)$. Con respecto a los valores de velocidad-agilidad se halló una correlación negativa con matemáticas $(\mathrm{r}=-0,605 ; \mathrm{p}=0,049)$, destrezas académicas $(\mathrm{r}=-0,670 ; \mathrm{p}=0,024)$ y rendimiento total $(\mathrm{r}=-0,706 ; \mathrm{p}=0,015)$. En este test también se observó una correlación negativa con tendencia a la significación con la lectura $(r=-0,581 ; p=0,061)$. 


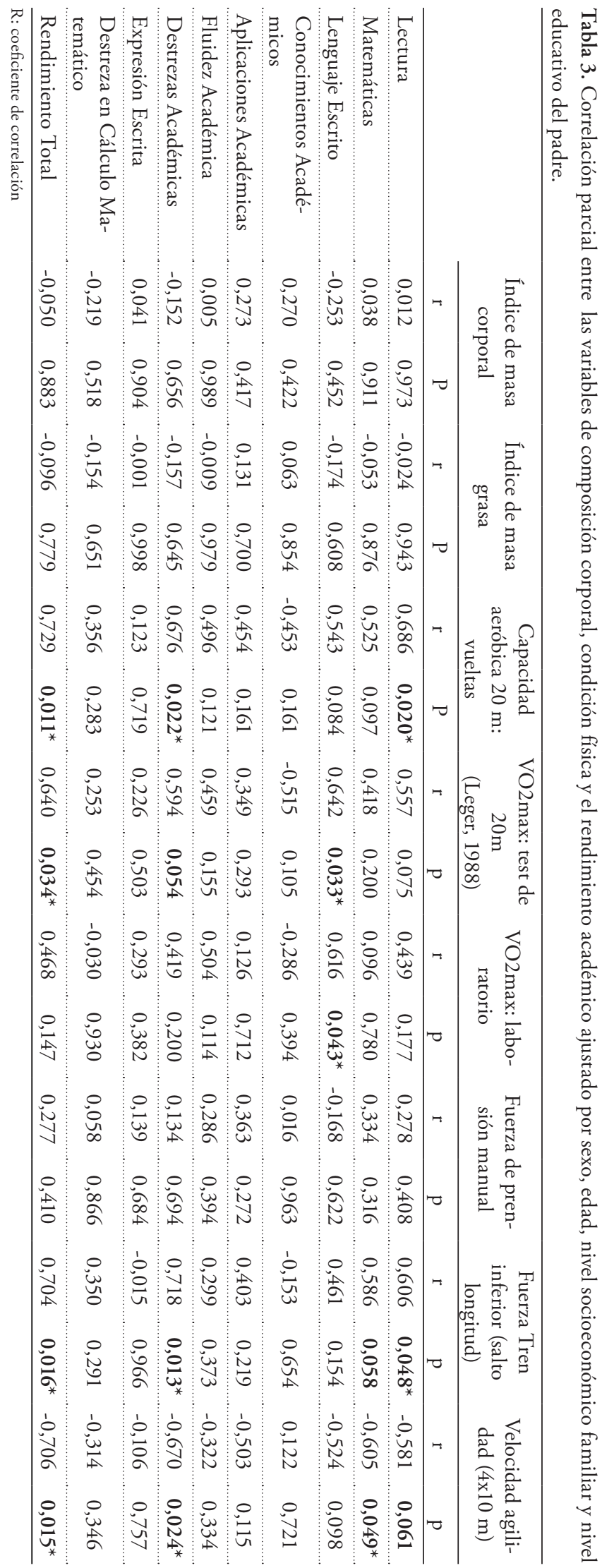


Se resaltan en letra negrita aquellas correlaciones que son significativas o con tendencia a la significación, con * si $\mathrm{p} \leq$ 0,05 , y sin símbolo si $0.05<\mathrm{p}>0,1$.

La tabla 4 muestra la regresión lineal entre los compuestos de rendimiento académico y variables de condición física. En lo que respecta a la capacidad aeróbica (test de $20 \mathrm{~m}$ ), se observó que por cada vuelta que se completó en el test, se producía una mejora en la puntuación de 1,58 en lectura, 1,16 en destrezas académicas y 1,15 en rendimiento total. En el VO${ }^{2}$ max medido con el test de ida y vuelta de $20 \mathrm{~m}$, se observó que por cada unidad de medida $(\mathrm{ml} / \mathrm{kg} / \mathrm{min})$ que se mejoraba, se producía una mejora del lenguaje escrito en 2,03 puntos, las destrezas académicas en 4,84 puntos y el rendimiento to- tal en 3,35 puntos. No obstante, para el $\mathrm{VO}^{2} \max$ medido en laboratorio, tan solo se producía una mejora del lenguaje escrito en 0,86 puntos por cada $\mathrm{ml} / \mathrm{kg} / \mathrm{min}$. Atendiendo a la fuerza del tren inferior (salto de longitud), se observó que por cada cm de mejora en el salto, se producía una mejora de 0,66 puntos en lectura, 0,36 puntos en matemáticas, 0,82 puntos en destrezas académicas y 0,52 puntos en rendimiento total. Por último, en la variable velocidad-agilidad, las relaciones son inversas, lo que significa que por cada segundo que se mejoraba el tiempo (menor tiempo) en la realización del test ( $4 \times 10 \mathrm{~m})$, se producía una mejora de la lectura en 7,89 puntos, en matemáticas, 4,68 puntos, en destrezas académicas 9,64 puntos y en rendimiento total 6,53 puntos.

Tabla 4. Regresión lineal entre los compuestos de rendimiento académico y variables de condición física.

\begin{tabular}{|c|c|c|c|c|c|c|}
\hline & & $\begin{array}{l}\text { Capacidad aeróbica } \\
20 \mathrm{~m} \text { : vueltas }\end{array}$ & $\begin{array}{c}\text { VO2max: test } \\
\text { de } 20 \mathrm{~m} \\
\text { (Leger, 1988) }\end{array}$ & $\begin{array}{l}\text { VO2max: } \\
\text { laboratorio }\end{array}$ & $\begin{array}{c}\text { Fuerza Tren } \\
\text { inferior (salto } \\
\text { longitud) }\end{array}$ & $\begin{array}{l}\text { Velocidad agilidad } \\
\qquad(4 \times 10 \mathrm{~m})\end{array}$ \\
\hline \multirow{3}{*}{ Lectura } & $\beta$ & 1,58 & - & - & 0,66 & $-7,89$ \\
\hline & IC $95 \%$ para $\beta$ & $(0,317-2,891)$ & - & - & $(0,007-1,313)$ & $(-16,231-0,437)$ \\
\hline & $\mathbf{P}$ & $0,020^{*}$ & - & - & $0,048^{*}$ & $0,061^{*}$ \\
\hline \multirow{3}{*}{ Matemáticas } & $\beta$ & - & - & - & 0,36 & $-4,68$ \\
\hline & IC $95 \%$ para $\beta$ & - & - & - & $(-0,015-0,744)$ & $(-9,347--0,031)$ \\
\hline & $\mathbf{P}$ & - & - & - & $0,058^{*}$ & $0,049^{*}$ \\
\hline \multirow{3}{*}{ Lenguaje Escrito } & $B$ & - & 2,03 & 0,86 & - & - \\
\hline & IC $95 \%$ para $\beta$ & - & $(0,204-3,869)$ & $(0,031-1,692)$ & - & - \\
\hline & $\mathrm{P}$ & - & $0,033^{*}$ & $0,043^{*}$ & - & - \\
\hline \multirow{3}{*}{ Destrezas Académicas } & $B$ & 1,16 & 4,84 & - & 0,82 & $-9,64$ \\
\hline & IC $95 \%$ para $\beta$ & $(0,297-3,025)$ & $(-0,101-9,787)$ & - & $(0,222-1,434)$ & $(-17,705--1,591)$ \\
\hline & $P$ & $0,022^{*}$ & $0,054^{*}$ & - & $0,013^{*}$ & $0,024^{*}$ \\
\hline \multirow{3}{*}{ Rendimiento Total } & $\beta$ & 1,15 & 3,35 & - & 0,52 & $-6,53$ \\
\hline & IC $95 \%$ para $\beta$ & $(0,337-1,965)$ & $(0,317-6,387)$ & - & $(0,124-0,919)$ & $(-11,472--1,599)$ \\
\hline & $\mathrm{P}$ & $0,011^{*}$ & $0,034^{*}$ & - & $0,016^{*}$ & $0,015^{*}$ \\
\hline
\end{tabular}

B, Beta no Estandarizada; IC, Intervalo de Confianza.

${ }^{*}$ Grado de significación a nivel $\mathrm{p} \leq 0,05$

\section{Discusión}

El presente estudio transversal examinó la asociación entre indicadores de masa corporal y masa grasa y condición física sobre el rendimiento académico en preadolescentes con sobrepeso/obesidad. Los principales hallazgos de este estudio muestran que la mayoría de las variables de condición física medidas (capacidad aeróbica en test de $20 \mathrm{~m}$, VO² max en test de $20 \mathrm{~m}$ y en laboratorio, fuerza del tren inferior, velocidadagilidad) tuvieron una relación positiva con el rendimiento académico. La fuerza del tren superior no mostró ninguna relación significativa. Así mismo no se hallaron relaciones significativas entre los parámetros de masa corporal y masa grasa con el rendimiento académico.

Los datos que muestra este estudio están en consonancia con otros estudios anteriores en lo que se refiere a la relación entre condición física, composición corporal y rendimiento académico. Un estudio de cohortes de Torrijos et al. (2014), con un total de 1531 niños/as $(9,49 \pm 0,71$ años) halló que la condición de sobrepeso se relaciona con puntuaciones de rendimiento académico más pobres. Otro estudio transversal de Davis y Cooper (2011) con un total de 170 niños/as de 7-11 ańos sedentarios con sobrepeso, relacionó el índice de masas grasa y el consumo de oxigeno con los compuestos de matemáticas y lectura del test Woodcock-Johnson III para el rendimiento académico, hallando relaciones entre el sobrepeso y los valores del rendimiento académico, no siendo estos resultados concluyentes.

Por otra parte, un estudio transversal de Castelli et al. (2007) realizado con 259 nińos/as $(9,5 \pm 0,74$ años) observa- 
ron la asociación entre la capacidad aeróbica y los test de fuerza muscular (flexiones y abdominales) con los compuestos de rendimiento total, matemáticas y lectura del rendimiento académico. El IMC también se relacionó de manera inversa. El estudio EDUFIT (Ardoy, 2014; Ardoy, 2011) de 16 semanas de duración con 67 adolescentes y basados en la duplicación de las horas de clase de educación física y en el aumento en la intensidad de éstas, arrojaron resultados de mejora de los parámetros de condición física evaluados como la capacidad aeróbica y flexibilidad como consecuencia del aumento del volumen de clases; el aumento de la intensidad produjo una mejora de la velocidad-agilidad y en el $\mathrm{VO}^{2} \max$ también se observaron mejoras en ambos casos. En cuanto al rendimiento académico, esta intervención también produjo mejoras en las puntuaciones de las materias de matemáticas, tecnología, ciencias naturales y educación física.

Estos resultados sugieren la hipótesis de que una mejora de la condición física en niños/as preadolescentes puede producir una mejora significativa en el rendimiento académico de éstos. Así, apoyándonos en estudios (Donnelly, 1996; Hills, 2011), relacionados con la práctica de actividad física como medio de mejora de la obesidad en niños/as, se puede pensar que como consecuencia de una mejora de la condición física, además del rendimiento académico, también se puede producir una mejora en los valores de composición corporal (masa corporal y masa grasa).

La principal limitación del presente estudio fue el tamaño tan pequeño de la muestra $(n=19)$. La consecución de un número más elevado de participantes, puede mostrar unos resultados más concluyentes e incluso mejores asociaciones entre las variables estudiadas. Igualmente, puede arrojar datos positivos sobre la asociación entre la obesidad y el rendimiento académico, confirmando así los hallazgos de estudios anteriores (Davis, 2011; Welk, 2010). Este, se trata de un estudio transversal, por lo que también se entiende que un programa de intervención, al igual que se realiza en los estudios anteriormente citados, supondría una mejora en la calidad de los datos obtenidos y en la relación de causalidad entre las variables de estudio.

En contraposición a esto, la principal ventaja del estudio se basó en la utilización de herramientas de alta precisión en la evaluación de las diferentes variables, como son el test de Woodcock-Muñoz para la valoración del rendimiento académico y el DXA para el índice de masa grasa. En el caso del DXA se puede hablar de "gold estándar". Un estudio de validación, comparando varios tipos de mediciones con el DXA en personas obesas, mostró la evidencia de la mayor precisión de éste (LaForgia, 2009). Por su parte, el test de aprovechamiento Woodcock-Muñoz viene siendo uno de los test más utilizados para la valoración del rendimiento académico, siendo muy extenso el número de compuestos de rendimiento que evalúa, así como la fiabilidad de sus puntuaciones. Los últimos y más importantes trabajos en relación a esto han utilizado esta batería de test. La batería ALPHA ha sido ampliamente validad, estudiada y el $\mathrm{VO}^{2}$ max, obtenido mediante una medida directa en el laboratorio, también se suman como una importante fortaleza del estudio.

Este estudios se centró en preadolescentes (9-11 años) con sobrepeso/obesidad, una futura línea de investigación puede ser la ampliación de las edades a estudiar. Otra posible línea de actuación podría estar centrada en establecer las relaciones de causalidad entre el rendimiento académico, la condición física y los motivos de abandono de la práctica deportiva en el entorno no escolar (escuelas deportivas, clubes deportivos, etc.).

\section{Conclusiones}

Como conclusión al estudio, se puede decir que altos niveles de condición física, en sus diferentes componentes relacionados con la salud (capacidad aeróbica, fuerza y velocidadagilidad) se asocian con mejores niveles de rendimiento académico, sin embargo, no hay una relación clara entre la masa grasa y el rendimiento académico. No obstante un estudio de intervención con un mayor tamaño de muestra confirmaría o contrastaría estos resultados y demostraría si las asociaciones observadas son causales o no.

\section{Implicaciones del estudio}

A menudo, muchos padres deciden no apuntar a sus hijos a actividades extraescolares, con el argumento de que necesitan más tiempo para hacer los deberes y estudiar. Sin embargo, los resultados del presente estudio, junto con los existentes en la literatura sugieren que realizar ejercicio físico que conlleve mejoras en la condición física podría relacionarse con mejoras en el rendimiento académico.

\section{Referencias}

1. Ardoy, D.N., Fernandez-Rodriguez, J.M., Jimenez-Pavon, D., Castillo, R., Ruiz, J.R., \& Ortega, F.B. (2014). A Physical Education trial improves adolescents' cognitive performance and academic achievement: the EDUFIT study. Scandinavian Journal of Medicine \& Science in Sports, 24(1), e52-e61.

2. Ardoy, D.N., Fernandez-Rodriguez, J.M., Ruiz, J.R., Chillon, P., Espa-
na-Romero, V., Castillo, M.J., et al. (2011). Improving Physical Fitness in Adolescents Through a School-Based Intervention: the EDUFIT Study. Revista Espanola De Cardiologia, 64(6), 484-491.

3. Castelli, D.M., Hillman, C.H., Buck, S.M., \& Erwin, H.E. (2007) Physical fitness and academic achievement in third- and fifth-grade students. Journal of Sport \& Exercise Psychology, 29(2), 239-252. 
4. Castillo, R., Ruiz, J.R., Chillon, P., Jimenez-Pavon, D., EsperanzaDiaz, L., Moreno, L.A., et al. (2011). Associations between parental educational/occupational levels and cognitive performance in Spanish adolescents: The AVENA study. Psicothema, 23(3), 349-355.

5. Cole, T.J., \& Lobstein, T. (2012). Extended international (IOTF) body mass index cut-offs for thinness, overweight and obesity. Pediatric Obesity, 7(4), 284-294.

6. Currie, C.E., Elton, R.A., Todd, J., \& Platt, S. (1997). Indicators of socioeconomic status for adolescents: the WHO Health Behaviour in School-aged Children Survey. Health Education Research, 12(3), 385397.

7. Davis, C.L., \& Cooper, S. (2011). Fitness, fatness, cognition, behavior, and academic achievement among overweight children: Do cross-sectional associations correspond to exercise trial outcomes?. Preventive Medicine, 52, S65-S69.

8. Davis, C.L., Pollock, N.K., Waller, J.L., Allison, J.D., Dennis, B.A., Bassali, R., et al. (2012). Exercise Dose and Diabetes Risk in Overweight and Obese Children A Randomized Controlled Trial. JAMA-J Am Med Assoc, 308(11), 1103-1012.

9. Donnelly, J.E., Jacobsen, D.J., Whatley, J.E., Hill, J.O., Swift, L.L., Cherrington, A., et al.(1996). Nutrition and physical activity program to attenuate obesity and promote physical and metabolic fitness in elementary school children. Obesity Research, 4(3), 229-243.

10. Donnelly, J.E., \& Lambourne, K. (2011). Classroom-based physical activity, cognition, and academic achievement. Preventive Medicine, 52, S36-S42.

11. Espańa-Romero, V., Garcia-Artero, E., Santaliestra-Pasias, A.M., Gutierrez, A., Castillo, M.J., \& Ruiz, J.R. (2008). Hand span influences optimal grip span in boys and girls aged 6 to 12 years. Journal of Hand Surgery-American Volume, 33A(3), 378-384.

12. Esteban-Cornejo, I., Tejero-Gonzalez, C.M., Martinez-Gomez, D., del-Campo, J., Gonzalez-Galo, A., Padilla-Moledo, C., et al. (2014). Independent and Combined Influence of the Components of Physical Fitness on Academic Performance in Youth. Journal of Pediatrics, 165(2), 306-312.

13. Etnier, J.L., Salazar, W., Landers, D.M., Petruzzello, S.J., Han, M., \& Nowell, P. (1997). The influence of physical fitness and exercise upon cognitive functioning: A meta-analysis. Journal of Sport \& Exercise Psychology, 19(3), 249-277.

14. Gracia-Marco, L., Ortega, F.B., Jimenez-Pavon, D., Rodriguez, G., Castillo, M.J., Vicente-Rodriguez, G., et al. (2012). Adiposity and bone health in Spanish adolescents. The HELENA study. Osteoporosis Int, 23(3), 937-947.

15. Hills, A.P., Andersen, L.B., \& Byrne, N.M. (2011). Physical activity and obesity in children. British Journal of Sports Medicine, 45(11), 866870 .

16. Jimenez-Pavon, D., Ortega, F.P., Ruiz, J.R., Espana-Romero, V., Garcia-Artero, E., Moliner-Urdiales, D., et al. (2010). Socioeconomic status influences physical fitness in European adolescents independently of body fat and physical activity: the HELENA study. Nutricion hospitalaria, 25(2), 311-316.
17. LaForgia, J., Dollman, J., Dale, M.J., Withers, R.T., \& Hill, A.M. (2009). Validation of DXA Body Composition Estimates in Obese Men and Women. Obesity, 17(4), 821-826.

18. Lees, C., \& Hopkins, J. (2013). Effect of aerobic exercise on cognition, academic achievement, and psychosocial function in children: a systematic review of randomized control trials. Preventing chronic disease, 10, E174.

19. Leger, L.A., Mercier, D., Gadoury, C., \& Lambert, J. (1988). The multistage 20 metre shuttle run test for aerobic fitness. Journal of sports sciences, 6(2), 93-101.

20. Ogden, C.L., Carroll, M.D., Kit, B.K., \& Flegal, K.M. (2014). Prevalence of Childhood and Adult Obesity in the United States, 2011-2012. JAMA-J Am Med Assoc, 311(8), 806-814.

21. Olson, R.K., Hulslander, J., Christopher, M., Keenan, J.M., Wadsworth, S.J., Willcutt, E.G., et al. (2013). Genetic and Environmental Influences on Writing and their Relations to Language and Reading. Annals of dyslexia, 63(1), 25-43.

22. Ortega, F.B., Ruiz, J.R., Castillo, M.J., \& Sjostrom, M. (2008). Physical fitness in childhood and adolescence: a powerful marker of health. International Journal of Obesity, 32(1), 1-11.

23. Pontifex, M.B., Raine, L.B., Johnson, C.R., Chaddock, L., Voss, M.W., Cohen, N.J., et al. (2011). Cardiorespiratory Fitness and the Flexible Modulation of Cognitive Control in Preadolescent Children. Journal of Cognitive Neuroscience, 23(6), 1332-1345.

24. Roberts, C.K., Freed, B., McCarthy, \& W.J. (2010). Low Aerobic Fitness and Obesity Are Associated with Lower Standardized Test Scores in Children. Journal of Pediatrics, 156(5), 711-718.

25. Ruiz, J.R., Castro-Pinero, J., Garcia-Artero, E., Ortega, F.B., Sjostrom, M., Suni, J., et al. (2009). Predictive validity of health-related fitness in youth: a systematic review. British Journal of Sports Medicine, 43(12), 909-923.

26. Ruiz, J.R., Castro-Pinero, J., Espana-Romero, V., Garcia-Artero, E., Ortega, F.B., Cuenca, M.M., et al. (2011). Field-based fitness assessment in young people: the ALPHA health-related fitness test battery for children and adolescents. British Journal of Sports Medicine, 45(6), 518-524.

27. Tanner, J.M., \& Whitehouse, R.H. (1976). Clinical longitudinal standards for height, weight, height velocity, weight velocity, and stages of puberty. Archives of disease in childhood, 51(3), 170-179.

28. Torrijos-Niño, C., Martinez-Vizcaino, V., Pardo-Guijarro, M.J., Garcia-Prieto, J.C., Arias-Palencia, N.M., \& Sanchez-Lopez, M. (2014). Physical Fitness, Obesity, and Academic Achievement in Schoolchildren. Journal of Pediatrics, 165(1), 104-109.

29. Utter, A.C., Robertson, R.J., Nieman, D.C., \& Kang, J. (2002). Children's OMNI scale of perceived exertion: Walking/running evaluation. Medicine and Science in Sports and Exercise, 34(1), 139-144.

30. Welk, G.J., Jackson, A.W., Morrow, J.R., Haskell, W.H., Meredith, M.D., \& Cooper, K.H. (2010). The Association of Health-Related Fitness With Indicators of Academic Performance in Texas Schools. Research Quarterly for Exercise and Sport, 81(3), S16-S23. 